\title{
POTENCIAL NUTRACEÚTICO DE LOS MAÍCES CRIOLLOS Y CAMBIOS DURANTE EL PROCESAMIENTO TRADICIONAL Y CON EXTRUSIÓN
}

\author{
NUTRACEUTICAL POTENTIAL OF NATIVE MAIZE AND CHANGES DURING \\ TRADITIONAL AND EXTRUSION PROCESSING
}

\author{
Sergio O. Serna-Saldívar', Janet A. Gutiérrez-Uribe ${ }^{1}$, \\ Saraid Mora-Rochin ${ }^{2}$ y Silverio García-Lara ${ }^{1 \star}$
}

\begin{abstract}
${ }^{1}$ Departamento de Biotecnología e Ingeniería de Alimentos, Instituto Tecnológico de Estudios Superiores de Monterrey (ITESM), Campus Monterrey, Tecnológico de Monterrey. 64849, Monterrey, Nuevo León. Tel.: +52 818358 1400; Fax: +52 818328 4262. Programa Regional de Posgrado en Alimentos, Universidad Autónoma de Sinaloa, Culiacán, Sinaloa.
\end{abstract}

*Autor para correspondencia (sgarcialara@itesm.mx)

\section{RESUMEN}

Una de las herencias de las culturas mesoamericanas se refleja en las variedades criollas de maíz (Zea mays L.) que se usan en México. Con excepción de la Antártida, hoy en día el maíz se cultiva en todos los continentes del mundo con una producción global que excede los 844 millones de toneladas. Las variedades criollas del maíz contienen genes que le imparten una variabilidad genética de suma importancia. Dentro de estas razas criollas existen accesiones con modificaciones en el color del grano y sus pigmentos, que le confieren propiedades fitoquímicas únicas. Primordialmente existen dos grandes familias de pigmentos asociados al maíz, los de naturaleza fenólica que incluyen a las antocianinas y los carotenoides. Los maíces ricos en carotenoides son ampliamente sembrados en el mundo entero, mientras que los ricos en antocianinas solamente se siembran en áreas muy limitadas. Los maíces ricos en antocianinas pueden ser considerados como nutracéuticos debido al efecto preventivo de estos pigmentos contra el estrés oxidativo, las enfermedades crónico-degenerativas y el cáncer. Otros importantes fitoquímicos son los fitoesteroles y policosanoles por sus probados efectos hipocolesterolémicos. Es importante resaltar que todos los maíces nativos contienen compuestos fenólicos de alto valor, de modo que los criollos constituyen un reservorio y patrimonio genético por explorar. Esta revisión describe a los principales fitoquímicos asociados al maíz, con énfasis en las variedades nativas, y además presenta las propiedades nutracéuticas de maíces criollos transformadas en tortillas mediante el procesamiento tradicional y con tecnologías emergentes como la extrusión, y su implicación en la alimentación y la salud.

Palabras clave: Zea mays, maíces criollos, compuestos fenólicos, antocianinas, carotenos, actividad antioxidante, nutracéuticos.

\section{SUMMARY}

One of the legacies of Mesoamerican cultures is reflected in maize (Zea mays L.) landraces varieties used in México. With the exception of Antarctica, maize is currently grown on all continents of the world, with global production exceeding 844 million tons. Maize landraces contain genes which impart genetic variability of utmost importance. Within these accessions there are maize landraces with modifications in grain color and pigments, which have unique phytochemical properties. There are two main families of pigments associated with corn, phenolicderived pigments, which include anthocyanins, and carotenoids. The carotenoid-rich maize is widely grown in the world, while the genotypes rich in anthocyanins are planted only in very limited areas.
Anthocyanin-rich corn can be considered as nutraceutical because these pigments have preventive effects against oxidative stress, chronic degenerative diseases and cancer. Other important phytochemical are phytosterols and policosanols which have hypocholesterolemic effects. Importantly, all native corn materials contain phenolic compounds of high value, so maize landraces constitute a reservoir and genetic heritage to be explored. This review describes the main phytochemicals associated with corn, with emphasis on native varieties, and also presents the nutraceutical properties of native corn tortillas processed by traditional processing and emerging technologies such as extrusion, and their involvement in food and health.

Index words: Zea mays, maize landraces, phenolic compounds, anthocyanins, carotenoids, antioxidant capacity, nutraceuticals.

\section{INTRODUCCIÓN}

Un mexicano consume diariamente un promedio de 335 g de maíz (Zea mays L.) lo que equivale a $122 \mathrm{~kg} / \mathrm{año}$ (FAO, 2012). Por tanto, si se considera una dieta de $2000 \mathrm{kcal}$, la población mexicana obtiene más de la mitad de las calorías y proteínas de alimentos producidos a partir del maíz (FAO, 2012). Las culturas indígenas prehispánicas han reconocido el valor de los maíces criollos desde tiempos remotos. Por ejemplo, la civilización azteca, la maya e inca relacionaron a los maíces pigmentados con el culto a deidades de la alimentación y subsistencia. La base culinaria de la cultura indígena Mesoamericana fue la gama de productos obtenidos principalmente del maíz nixtamalizado (cocido con calcio). Los indígenas aprendieron a cocinar las cariópsides en cenizas de hoguera o hidróxido de calcio o cal obtenida de conchas o de depósitos mineros (Serna-Saldívar, 2009).

Durante la nixtamalización, el almidón del maíz se pregelatiniza, la proteína se desnaturaliza y el pericarpio o salvado se hidroliza parcialmente. Nutrimentalmente, el maíz cocido o nixtamal contiene una alta cantidad de calcio biodisponible y mejor disponibilidad de la niacina. En la mayoría de los casos el nixtamal es lavado y posteriormente molido para obtener una masa que es la columna vertebral para la 
generación de numerosos alimentos típicos ancestrales, y que todavía son la base de la alimentación de los mexicanos. El icono de los alimentos nixtamalizados es la tortilla, que ha sido producida a partir de numerosos tipos de maíces (blanco, amarillo, azul y morado) (Serna-Saldívar, 2009).

Los maíces criollos actuales poseen una gran diversidad genética y potencial para producir alimentos funcionales que ayuden a la población más vulnerable a enfrentar los graves problemas de desnutrición y salud. A través de generaciones los maíces han conservado importantes compuestos fitoquímicos conocidos como nutracéuticos, con probados efectos positivos en nutrición y salud, como: fibra dietética (soluble e insoluble), compuestos fenólicos, carotenoides, xantofilas, triglicéridos ricos en ácidos grasos omega 6, fitoesteroles, policosanoles y micronutrientes como tocoferoles y tocotrienoles, fosfolípidos que proveen colina e inositol, y vitaminas con propiedades nutracéuticas como el ácido fólico, tiamina y niacina. El común denominador de la mayoría de los nutracéuticos es su capacidad antioxidante que contrarresta los radicales libres responsables de causar la oxidación de membranas y daño al ADN, lo que promueve enfermedades como cáncer, fibrosis, problemas cardiovasculares y envejecimiento (Serna-Saldívar, 2009; Serna-Saldívar et al., 2011). El procesamiento del maíz con diferentes perfiles nutracéuticos afecta significativamente la cantidad y biodisponibilidad de estos compuestos. Por ejemplo, el proceso de nixtamalización propicia que parte de la fibra dietética y muchos compuestos fenólicos simples y antocianinas se pierdan en la cocción alcalina, pero los compuestos que quedan asociados al nixtamal tienen una mejor biodisponibilidad (Serna-Saldívar, 2009). La molienda en seco del maíz generalmente remueve al salvado y al germen para obtener fracciones refinadas del endospermo, remoción que disminuye significativamente el contenido de fitoquímicos, vitaminas y minerales.

\section{EL ALTO VALOR NUTRACÉUTICO DE LOS MAÍCES CRIOLLOS}

México es considerado como el centro de origen y diversidad del maíz. Esta diversidad se ha agrupado en al menos 59 razas con base en características morfológicas, bioquímicas, genéticas y moleculares (Warburton et al., 2008). Como variantes dentro de las razas se ha propuesto una clasificación por tipos o por coloración de grano. El color se determina por el contenido de pigmentos encontrados, como los carotenoides en los granos amarillos y las antocianinas y flobafenos en los azules o rojos (Egesel et al., 2003). Estas variedades pigmentadas están presentes en 41 razas, ya sea por su color en el grano o bien por la presencia de antocianinas en hojas, tallo y mazorca (Ortega et al., 1991). Si bien el objetivo primordial del mejoramiento genético del maíz es el rendimiento, recientemente se ha puesto mayor énfasis en el desarrollo de variedades con composiciones químicas nutrimentales diferenciadas y con alta concentración de compuestos nutracéuticos (Serna Saldívar, 2010).

Los compuestos nutracéuticos asociados con el maíz no son necesariamente nutrientes que tradicionalmente han sido asociados con deficiencias en la dieta, sino compuestos cuyo consumo ayuda a prevenir e inclusive tratar a las enfermedades crónico-degenerativas. Estos compuestos se clasifican de acuerdo con sus propiedades químicas y su actividad biológica. Conviene destacar que la diversidad presente entre y dentro de las razas de maíces criollos juega un papel fundamental que incluye la presencia de compuestos nutraceúticos. Aunque aún no se ha determinado por completo qué maíces criollos son mejores, en el Cuadro 1 se presenta un resumen de los principales compuestos nutracéuticos asociados con los diferentes tipos de maíces criollos.

\section{COMPOSICIÓN FITOQUÍMICA Y PROPIEDADES NUTRACÉUTICAS}

Las propiedades nutracéuticas del maíz están íntimamente ligadas con su composición fitoquímica, y representan actualmente una línea de investigación de gran interés científico, donde los maíces criollos constituyen una fuente de diversidad fenotípica y genotípica por explorar, porque contiene gran cantidad de compuestos con relevantes propiedades nutracéuticas (Cuadro 1).

El grano de maíz produce diversos tipos de compuestos fenólicos que son categorizados como simples, flavonoides y antocianinas. Todos los maíces contienen fenólicos simples, mientras que solamente los maíces pigmentados como el azul o morado contienen cantidades significativas de antocianinas. El ácido ferúlico es el compuesto fenólico presente en mayor cantidad en maíz, donde se le puede encontrar en forma ligada, libre y condensada. Otro importante grupo de antioxidantes son los carotenoides y xantofilas. Adicionalmente, los $\beta$-carotenos son convertidos en la forma activa de la vitamina A o retinol, considerada como la de mayor relevancia en nutrición humana porque previene la ceguera total y nocturna, y otras enfermedades como xeroftalmia, cáncer, enfermedades cardiovasculares, y también ayuda a reforzar el sistema inmune. Las xantofilas luteína, zeaxantina y criptoxantina ayudan a prevenir la degeneración macular asociada con la ceguera, especialmente en adultos mayores. El maíz amarillo es rico en carotenos y xantofilas, en contraste con el blanco que contiene cantidades insignificantes de estos importantes nutracéuticos (Serna-Saldívar et al., 1990; Serna-Saldívar, 2009; 2012). 
Cuadro 1. Principales categorías de nutracéuticos asociados con maíces criollos ${ }^{\dagger}$.

\begin{tabular}{|c|c|c|c|}
\hline \multicolumn{2}{|c|}{ Compuesto nutracéutico } & \multirow{2}{*}{ Estructura anatómica } & \multirow{2}{*}{ Efecto preventivo o terapéutico } \\
\hline Familia & Clase & & \\
\hline Fenólicos & $\begin{array}{l}\text { Simples como el } \\
\text { ferúlico }\end{array}$ & $\begin{array}{l}\text { Principalmente asociados } \\
\text { al pericarpio. }\end{array}$ & $\begin{array}{l}\text { Previenen el estrés oxidativo, cáncer, alto colesterol, } \\
\text { arterioesclerosis y envejecimiento. }\end{array}$ \\
\hline $\begin{array}{l}\text { Antocianinas y flavo- } \\
\text { noides }\end{array}$ & $\begin{array}{l}\text { Antocianinas, flavo- } \\
\text { noles, flavan-3-ols }\end{array}$ & $\begin{array}{l}\text { Principalmente asociados } \\
\text { a la aleurona. }\end{array}$ & $\begin{array}{l}\text { Previenen el estrés oxidativo, cáncer, alto colesterol, } \\
\text { arterioesclerosis y envejecimiento. }\end{array}$ \\
\hline \multirow[b]{2}{*}{ Carotenoides } & Carotenos & $\begin{array}{l}\text { Principalmente asociados } \\
\text { al endospermo de maíces } \\
\text { amarillos. }\end{array}$ & $\begin{array}{l}\text { Los B-carotenos son convertidos en vitamina A o reti- } \\
\text { nol. Previenen el cáncer, las enfermedades cardiovascu- } \\
\text { lares y refuerzan al sistema inmunológico. }\end{array}$ \\
\hline & $\begin{array}{l}\text { Xantofilas: } \\
\text { luteína, zeaxantina, } \\
\text { criptoxantina }\end{array}$ & $\begin{array}{l}\text { Principalmente asociados } \\
\text { al endospermo de maíces } \\
\text { amarillos. }\end{array}$ & $\begin{array}{l}\text { Previenen a la degradación macular y formación de } \\
\text { cataratas (opacidad del cristalino ocular). Retarda apa- } \\
\text { rición de síntomas relacionados con la retinitis. Previe- } \\
\text { nen a las enfermedades cardiovasculares. }\end{array}$ \\
\hline Fitoesteroles & $\begin{array}{l}\text { Sitosterol, estigmas- } \\
\text { terol, campesterol }\end{array}$ & $\begin{array}{l}\text { Principalmente asociados } \\
\text { al germen, pericarpio y } \\
\text { aleurona }\end{array}$ & $\begin{array}{l}\text { Compiten con los sitios de absorción del colesterol y por } \\
\text { consiguiente son considerados como hipocolesterolé- } \\
\text { micos y previsores de enfermedades cardiovasculares. }\end{array}$ \\
\hline \multirow[t]{2}{*}{ Fibra } & $\begin{array}{l}\text { Soluble como arabi- } \\
\text { noxilanos y hemice- } \\
\text { lulosas solubles. }\end{array}$ & $\begin{array}{l}\text { Principalmente asociada } \\
\text { al pericarpio. }\end{array}$ & $\begin{array}{l}\text { Mejora función gastrointestinal, incrementa la visco- } \\
\text { sidad del bolo alimenticio y reduce el índice glicémico. } \\
\text { Reduce el riesgo de diabetes e hipercolesterolemia. Efec- } \\
\text { to prebiótico generando ácidos grasos volátiles. }\end{array}$ \\
\hline & $\begin{array}{l}\text { Insoluble como celu- } \\
\text { losa y lignina }\end{array}$ & $\begin{array}{l}\text { Principalmente asociadas } \\
\text { al endospermo de maíces } \\
\text { amarillos. }\end{array}$ & $\begin{array}{l}\text { Mejora función gastrointestinal, incrementa el ligado } \\
\text { de ácidos biliares y el volumen y peso de las heces, y re- } \\
\text { duce constipación, hemorroides, diverticulosis y cáncer. }\end{array}$ \\
\hline Ácido fítico inositol & Inositol & $\begin{array}{l}\text { Principalmente asociado } \\
\text { al pericarpio y aleurona. }\end{array}$ & $\begin{array}{l}\text { Considerado como antioxidante y con propiedades an- } \\
\text { ticancerígenas de mama, colon, hígado, leucemia, prós- } \\
\text { tata y sarcomas. }\end{array}$ \\
\hline $\begin{array}{l}\text { Ácidos grasos polin- } \\
\text { saturados }\end{array}$ & $\begin{array}{l}\text { Ácidos linoleico } \\
(18: 2 \omega 6) \text { y linoléni- } \\
\text { co }(18: 3 \omega 3)\end{array}$ & $\begin{array}{l}\text { Principalmente asociada } \\
\text { al germen. }\end{array}$ & $\begin{array}{l}\text { Ayudan a reducir triglicéridos y colesterol sanguíneo. } \\
\text { Estos ácidos grasos son convertidos en prostaglandinas } \\
\text { y otros ácidos grasos como EPA y DHA. }\end{array}$ \\
\hline Fosfolípidos & $\begin{array}{l}\text { Fosfatidil colina, } \\
\text { fosfatidil etanolami- } \\
\text { na, fosfatidil inositol, } \\
\text { fosfatidil serina }\end{array}$ & $\begin{array}{l}\text { Principalmente asociados } \\
\text { al germen. }\end{array}$ & $\begin{array}{l}\text { Esenciales para el funcionamiento apropiado de las } \\
\text { membranas celulares y de las neuronas cerebrales. Pre- } \\
\text { vienen el envejecimiento celular y el alto colesterol. La } \\
\text { colina es esencial para producir acetil colina considera- } \\
\text { da como el principal neurotransmisor. }\end{array}$ \\
\hline \multirow[t]{2}{*}{ Vitaminas } & Tocoferoles & $\begin{array}{l}\text { Principalmente asociados } \\
\text { al germen. }\end{array}$ & $\begin{array}{l}\text { Los tocoferoles son antioxidantes. El } \alpha \text {-tocoferol o vita- } \\
\text { mina E es considerado como la segunda línea de defen- } \\
\text { sa contra el estrés oxidativo que previene enfermedades } \\
\text { cardiovasculares, alto colesterol y todo tipo de cáncer. } \\
\text { Además ayudan a mejorar la capacidad mental y fun- } \\
\text { ción cerebral. }\end{array}$ \\
\hline & Ácido fólico & $\begin{array}{l}\text { Principalmente asociados } \\
\text { a la capa de aleurona. }\end{array}$ & $\begin{array}{l}\text { Previene abortos y los defectos de tubo neural y mal- } \\
\text { formaciones en bebés. Ayuda a reducir los niveles de } \\
\text { homocisteína y por ello previene enfermedades cardio- } \\
\text { vasculares. }\end{array}$ \\
\hline Policosanoles o ceras & $\begin{array}{l}\text { Octacosanol, tricon- } \\
\text { tanol, hexacosanol, } \\
\text { dotriacontanol }\end{array}$ & $\begin{array}{l}\text { Principalmente asociados } \\
\text { al germen y pericarpio. }\end{array}$ & $\begin{array}{l}\text { Reducen lípidos séricos, colesterol y la agregación de } \\
\text { plaquetas }\end{array}$ \\
\hline
\end{tabular}


La fibra dietética es uno de los constituyentes más importantes de la dieta debido a que el hombre moderno no la consume en cantidad suficiente, sino aproximadamente la mitad de la dosis recomendada. Los granos integrales, que incluyen al maíz, aportan cantidades significativas de fibra dietética, especialmente la insoluble, que mejora la función gastrointestinal y previene constipación, divertículos, hemorroides, cáncer de colon y diabetes. Adicionalmente, el maíz es rico en fitatos, que si bien tradicionalmente han sido considerados como factores antinutrimentales porque disminuyen la biodisponibilidad de minerales como el hierro, pero que también poseen propiedades anticarcinogénicas (Steer y Gibson, 2002).

El germen del maíz, que contiene más de $85 \%$ de los lípidos asociados con el grano entero, contiene además cantidades importantes de triglicéridos ricos en ácidos linoleico y oleico, y de fosfolípidos como fosfatidil colina, fosfatidil inositol, fosfatidil etanolamina y fosfatidil serina. Los fosfolípidos ayudan a mantener una buena integridad de las membranas celulares y un adecuado funcionamiento cerebral. En adición, el aceite crudo de maíz contiene cantidades relevantes de derivados de tocoles, como tocoferoles y tocotrienoles que son responsables de la actividad de vitamina E (Chung y Ohm, 2000), sustancias consideradas como el segundo más importante mecanismo de defensa contra radicales libres y estrés oxidativo.

Estos potentes antioxidantes previenen la oxidación de lípidos, ácidos grasos poli-insaturados y del colesterol LDL (lipoproteínas de baja densidad, por su siglas en inglés), cuya acumulación exacerba las enfermedades cardiovasculares (Serna-Saldívar, 2010). El maíz contiene de 0.03 a $0.33 \%$ de tocoferoles en su aceite, y sus formas $\alpha-y$ $\gamma$-tocoferol son las más abundantes (White y Weber, 2003). La principal función de estos compuestos es prevenir el estrés oxidativo y la oxidación del ácido linoleico $(18: 2 \Delta 9,12)$. Kornfeldt y Croon (1981) evaluaron el contenido de cuatro tipos de maíz (dos normales -no ricos en aceite ni en lisina-, uno alto en aceite, y uno clasificado como opaco 2 con alto contenido del aminoácido lisina), y concluyeron que entre 70 y $80 \%$ de los tocoferoles se encuentran asociados al germen y de 11 a $27 \%$ al endospermo. Tal como se esperaba, el genotipo rico en aceite presentó las mayores cantidades de tocoferoles $\alpha$ y $\gamma$.

El maíz es rico en fitoesteroles como sitoesterol, campesterol y estigmaesterol. De acuerdo con Kornfeldt y Croon (1981), el aceite de maíz contiene 1441, 62 y $54 \mathrm{mg} / 100 \mathrm{~g}$, de los esteroles 4 desmetilesterol, 4-monometilesterol y 4,4 dimetilesterol, respectivamente. Entre los desmetilesteroles, el sitosterol es la forma predominante (60 a $70 \%$ ), seguido por campesterol (16 a $22 \%$ ) y estigmaesterol (4 a $10 \%$ ). Debido a que estos compuestos tienen una estructura similar al colesterol presente en tejidos animales, el consumo de fitoesteroles inhibe la absorción del colesterol en las células epiteliales del intestino.

Por tanto, los fitoesteroles son compuestos hipocolesterolémicos que ayudan a prevenir los padecimientos cardiovasculares. Se ha establecido que el consumo diario de 1 a $3 \mathrm{~g}$ de fitoesteroles reduce el colesterol sanguíneo en 5 a 20 $\%$. Cabe mencionar que el consumo promedio diario es entre 200 a $400 \mathrm{mg}$ (White y Weber, 2003). De acuerdo con Raicht et al. (1980) y Fiala et al. (1985), la suplementación de $0.2 \%$ de $\beta$-sitoesterol en dietas para animales de laboratorio causó una disminución significativa en la incidencia de tumores cancerígenos de colon inducidos químicamente. Aún no se ha estudiado qué les sucede a los fitoesteroles durante los pasos secuenciales de la nixtamalización para la producción de tortillas. Dado que en este proceso se pierden parcialmente los tejidos del pericarpio y germen, es de esperarse que la nixtamalización afecte significativamente las cantidades y biodisponibilidad de estos importantes compuestos.

El maíz también es fuente importante de compuestos cerosos conocidos como policosanoles, que son ceras principalmente asociadas al epicarpio y germen. Los policosanoles consisten de una mezcla de alcoholes de cadena larga, como hexacosanol (26:0), octacosanol (28:0), triacontanol (30:0) y docatriacontanol (32:0). De acuerdo con Hwang et al. (2005), los maíces contienen aproximadamente 10 mg/100 g de policosanoles. Según Arruzazabala et al. (1994, 1996), los policosanoles reducen los niveles de lípidos en sangre así como la agregación plaquetaria relacionada con enfermedades cardiovasculares.

Los platillos tradicionales elaborados con granos pigmentados de maíz, como atoles, pinoles, tortillas y frituras pigmentadas, presentan diversas tonalidades de color, desde rosa hasta azul intenso y un contenido adecuado de antocianinas y fenoles, por lo que resultan ser productos funcionales con buen potencial para el mercado (El-Sayed et al., 2006), además de que las tortillas son la principal forma de consumo de maíz en México y Centro América (SernaSaldívar et al., 1990; 2000).

Todo esto se ve complementado por la alta capacidad antioxidante de los granos de maíz, que es superior a la de otros cereales como el trigo (Triticum aestivum L.), avena (Avena sativa L.) y arroz (Oryza sativa L.) (De la Parra et al., 2007; Adom y Lui, 2002). De manera particular, el maíz pigmentado contiene altos niveles de antocianinas y otros fenólicos con una excelente capacidad antioxidante (Betran et al., 2001; Harrigan et al., 2007). En experimentos con ratones de laboratorio alimentados con antocianinas de maíz morado, particularmente con la cianidina-3-glucósido, 
Tsuda et al. (2003) encontraron que la dieta hiperlipidémica rica en antocianinas produjo menor ganancia de peso en los ratones y redujo significativamente la aparición de enfermedades como hiperglicemia, hiperinsulinemia (resistencia a la insulina) e hiperleptinemia, en comparación con el grupo alimentado con una dieta hiperlipidémica sin antocianinas; estos autores concluyeron que las antocianinas se pueden considerar como un aditivo funcional que previene la obesidad, la diabetes y el síndrome metabólico.

\section{Actividad antioxidante}

La capacidad antioxidante de los cereales es mayoritariamente debida a los compuestos fenólicos. Según De la Parra et al. (2007), los diferentes tipos de maíz contienen de 41 a 49.6 moles equivalentes de vitamina $\mathrm{C} / 100 \mathrm{~g}$. El maíz rico en carotenos presentó la mayor actividad antioxidante, ya que la capacidad antioxidante hidrofílica representó $98 \%$ de la capacidad antioxidante total. Sorprendentemente, el maíz azul presentó actividad antioxidante similar a la de los maíces blancos o amarillos, como mostraron Del PozoInsfran et al. (2006) en dos genotipos de maíces azules cuya actividad (25 a $29 \mu \mathrm{mol}$ equivalentes Trolox/g) superó al maíz blanco $(17.5 \mu$ mol equivalentes Trolox/g). Recientemente, Mora-Rochín et al. (2010) determinaron que la capacidad antioxidante de maíces blancos, amarillos, azules y rojos fue de 19.4, 19.1, 12.4 y $19.2 \mu$ mol equivalentes de Trolox/g, respectivamente.

Las antocianinas tienen la habilidad de capturar radicales libres como el superóxido $\left(\mathrm{O}_{2}\right)$, oxígeno $\left(\mathrm{O}_{2}\right)$, peróxido (ROO-), peróxido de hidrógeno $\left(\mathrm{H}_{2} \mathrm{O}_{2}\right)$ y radicales de hidroxilo $\left(\mathrm{OH}^{-}\right)$. La acción antioxidante de las antocianinas es atribuida específicamente a la presencia de grupos hidroxilos en la posición 3 del anillo $\mathrm{C}$ y en las posiciones 3', 4' y 5' del anillo B (Cone, 2007). En general, la capacidad antioxidante de las antocianidinas (agliconas) es superior al de antocianinas, capacidad que se incrementa al aumentar el número de azúcares en la molécula.

Los efectos antioxidantes de las antocianinas in vitro se han demostrado en varios cultivos celulares de células de colon, endoteliales, hepáticas y leucocitos. En dichos sistemas las antocianinas han exhibido efectos anti-tóxicos y anti-carcinogénicos, como: destrucción de radicales libres, reducción de la capacidad de absorción de radicales libres de las células, estimulación de enzimas de fase II para la desintoxicación, y reducción de varios procesos negativos, como la formación de productos oxidativos en el ADN, peroxidación de lípidos, mutagénesis ocasionada por toxinas ambientales y carcinógenos, y la proliferación celular por medio de la modulación de vías de señalización (Wang y Stoner, 2008).
En sus estudios, Shih et al. (2005) demostraron la habilidad de las antocianinas en la inducción de enzimas de fase II en células hepáticas de rata alimentadas con $50 \mu \mathrm{M}$ de antocianinas, y la formación de células no cancerosas de mama con una dieta de 10 a $20 \mu \mathrm{g} \mathrm{mL}^{-1}$ de antocianinas. Las antocianinas ocasionaron un incremento antioxidante mediante la activación de la enzima mas importante en el sistema fase II, la quinona reductasa, y activaron a las enzimas glutatión reductasa, glutatión peroxidasa y glutatión S-transferasa.

\section{Actividad antimutagénica}

Los granos de maíz contienen una compleja mezcla de componentes, algunos con propiedades antimutagénicas (Pedreschi et al., 2007). Un extracto de maíz azul mostró propiedades antimutagénicas al inhibir la carcinogénesis colo-rectal en ratas macho F344 tratadas con 1,2-dimetilhidrazina (Hagiwara et al., 2001; Pedreschi et al., 2007). La inhibición se atribuyó a las antocianinas presentes en el maíz azul, aunque no se descartó la posible acción de otros compuestos fenólicos y flavonoides en el extracto de maíz. Acciones antimutagénicas contra el mutágeno Trp-P-1 presentaron una respuesta de $96 \%$ para las fracciones de maíz azul a una concentración de $850 \mu \mathrm{g} \mathrm{mL}^{-1}$. La actividad antioxidante probada fue de $0.954 \mu \mathrm{g}$ equivalentes de Trolox, valor que representa una actividad antioxidante más alta que la de arándano (Vaccinium corymbosum). Las fracciones revelaron la presencia de formas glucosidadas de cianidina, peonidina, pelargonidina, y sus contrapartes aciladas. Se ha concluido que el potencial antimutagénico se debió a antocianinas glucosidadas y en menor grado a antocianinas aciladas (Pedreschi et al., 2007).

\section{Actividad quimiopreventiva}

Los compuestos antioxidantes se pueden clasificar en dos tipos, de acuerdo con su mecanismo de acción celular: inductores bifuncionales y monofuncionales. Los primeros aumentan las enzimas involucradas en la respuesta de enzimas anti-oxidantes en su fase I (glutatión-S-transferasa) y fase II (quinona reductasa, QR). Los segundos afectan la actividad primordial de enzimas fase II, sin efecto significativo en las enzimas de fase I. Las enzimas fase II generan un efecto fundamental de quimio-prevención (Prochaska y Talalay, 1988). Un estudio reciente de López-Martínez et al. (2011) demostró que los maíces con altos contenidos de antocianinas inducen actividad de la QR en líneas celulares de Hepatoma murino (Hepa $1 \mathrm{clc} 7$ ), pero que esta actividad se reduce significativamente cuando el maíz es sometido a nixtamalización. 


\section{IMPACTOS DURANTE EL PROCESAMIENTO}

\section{Cambios fitoquímicos y nutracéuticos}

La población mexicana, con más de 116 millones de personas, consume anualmente más de 11 millones de toneladas de maíz en forma de tortillas y otros productos nixtamalizados. Se estima que de cada diez tortillas, siete son elaboradas mediante procesos tradicionales e industriales de masa fresca, y solamente tres son producidas a partir de harina nixtamalizada. De acuerdo con la Cámara Nacional del Maíz Industrializado, el consumo anual promedio de tortilla para toda la población nacional se mantiene en el orden de $105 \mathrm{~kg}$ de maíz por persona. En promedio, los mexicanos obtienen aproximadamente la mitad de la ingesta calórica de los productos nixtamalizados.

La nixtamalización causa cambios relevantes en el perfil nutracéutico del maíz. Del Pozo-Insfran et al. $(2006,2007)$ estudiaron el destino de los polifenoles y compuestos relacionados durante la cocción alcalina para tortillas y frituras de maíces azules. Granos comerciales de color blanco y azul (tanto mexicano como americano) fueron secuencialmente procesados a masa, tortillas y frituras de maíz. El criollo mexicano azul presentó mayor concentración de antocianinas $(13.9 \mathrm{mg} / 100 \mathrm{~g})$, polifenoles $(5 \mathrm{mg} / 100 \mathrm{~g})$ y capacidad antioxidante (3.4 $\mu \mathrm{M}$ equiv. Trolox $/ \mathrm{mg}$ ), que el maíz azul americano. Las pérdidas de antocianinas en los procesos de elaboración de la masa, tortillas y frituras fueron de 39, 53 y $78 \%$, respectivamente. De la Parra et al. (2007) también determinaron la cantidad de fenólicos totales, ácido ferúlico, antocianinas y actividades antioxidantes hidrofílica y lipofílica de cinco tipos de maíz procesados en masa, tortilla y fritura de tipo totopo (Cuadro 2). Sus resultados demostraron que el proceso alcalino redujo significativamente el contenido de fenólicos totales y la capacidad antioxidante; los niveles observados de fenólicos totales en el grano (244 a $320 \mathrm{mg}$ ácido gálico/100 g base seca, bs), masa (125 a $198 \mathrm{mg}$ ácido gálico/100 g bs), tortilla (137 a 207 mg ácido gálico/100 g bs) y totopos (111 a $155 \mathrm{mg}$ ácido gálico/100 $\mathrm{g}$ bs), indicaron claramente la reducción significativa en estos importantes fitoquímicos.

El maíz azul sin procesar contuvo las cantidades mayores de antocianinas (36.9 mg equiv. cianidina-3 glucósido/100 $\mathrm{g}$ bs), seguido por el maíz rojo $(9.8 \mathrm{mg}$ ) y el resto de los genotipos estudiados (0.6 a $4.6 \mathrm{mg}$ ). Desafortunadamente, aproximadamente $80 \%$ de las antocianinas del maíz azul se perdieron durante la cocción alcalina. De acuerdo con Salinas-Moreno et al. (2003), la mayoría de las antocianinas se degradan durante la nixtamalización. Del Pozo-Insfran et al. $(2006,2007)$ también concluyeron que el maíz blanco no contiene cantidades detectables de antocianinas, y confirmaron que el tratamiento alcalino tiene un impacto negativo en la estabilidad de los compuestos fenólicos, antocianinas y capacidad antioxidante.

Recientemente, Gutiérrez-Uribe et al. (2010) recuperaron el "nejayote" (residuo líquido de aspecto lechoso derivado de la cocción alcalina del maíz,) generado durante la nixtamalización de diversos maíces. En los sólidos del "nejayote" encontraron concentraciones de 28.2 a 39.5 y de 203.6 a $246.6 \mathrm{mg}$ equivalentes de ácido gálico/100g bs de fenólicos libres y ligados, respectivamente. Los diversos sólidos de "nejayote" tenían de 10 a 100 veces más de ácido ferúlico libre que los granos originales. Similarmente, los sólidos del "nejayote" presentaron aproximadamente 20 veces más ácido ferúlico ligado, en comparación con los granos originales o la masa.

Las actividades antioxidantes producidas por compuestos ligados a grano, masa y "nejayote" fueron 5, 3.2 y 24 veces más altos que los comparados con la ejercida por los compuestos libres. Las actividades antioxidantes de los compuestos ligados asociados al "nejayote" fueron 193 y 60 veces mayores que los observados en grano crudo y en masa, respectivamente. Estas dramáticas diferencias se atribuyen a la cocción alcalina y a la lixiviación de los compuestos fenólicos y antioxidantes asociados principalmente al pericarpio y capa de aleurona. Por consiguiente, los sólidos del "nejayote" pueden ser utilizados para la extracción de antioxidantes y compuestos nutracéuticos.

Respecto al grupo de carotenos, es necesario tener en cuenta que hay dos grandes tipos de carotenoides: carotenos y xantofilas, y ambos son responsables de impartir el color amarillo típico al endospermo de los maíces amarillos. Los carotenos son precursores de la vitamina A, y las xantofilas luteína, zeaxantina y criptoxantina son nutracéuticos que previenen la degeneración macular, las enfermedades cardiovasculares y el cáncer (Serna-Saldívar, 2010). El grano de maíz contiene de 0.1 a $77 \mathrm{mg}$ de carotenos totales/ $\mathrm{kg}$, y los maíces blancos y amarillos contienen 0 y $30 \mathrm{mg} /$ $\mathrm{kg}$ de carotenos totales, respectivamente. Aproximadamente $95 \%$ de los carotenos se encuentran en el endospermo. Luteína y zeaxantina son las formas predominantes de las xantofilas (White y Weber, 2003).

Las pérdidas de estos pigmentos durante la nixtamalización fueron primeramente estudiadas por Bressani et al. (1958), quienes reportaron pérdidas de 26 a $34.5 \%$ del total de los carotenoides originalmente encontrados en un maíz amarillo. De la Parra et al. (2007) compararon a los carotenos de cinco tipos de maíz y sus productos nixtamalizados (Cuadro 3). La mayor concentración de luteína se observó en el maíz amarillo ( $406.2 \mu \mathrm{g} / 100 \mathrm{~g}$ bs) y la más baja en los maíces azul y blanco $(5.2$ y $5.7 \mu \mathrm{g} / 100 \mathrm{~g}$ bs, respectivamente). La cocción alcalina ocasionó importantes pérdidas de 
Cuadro 2. Efecto de la nixtamalización en los perfiles fitoquímicos de maíces criollos blanco, amarillo, rojo, azul y mejorado alto en carotenos, procesados en tortillas y totopos ${ }^{\dagger}$.

\begin{tabular}{|c|c|c|c|c|c|c|}
\hline \multirow{2}{*}{ Tipo de maíz/producto ${ }^{\dagger \dagger}$} & \multicolumn{2}{|c|}{ Fenoles totales } & \multicolumn{3}{|c|}{ Ácido ferúlico } & \multirow{2}{*}{ Antocianinas } \\
\hline & Libre & Ligado & Libre & Conjugado & Ligado & \\
\hline Grano & \multicolumn{2}{|c|}{$\begin{array}{l}\text { (mg equivalentes ácido } \\
\text { gálico/100 g) }\end{array}$} & \multicolumn{3}{|c|}{$(\mu \mathrm{g} / 100 \mathrm{~g})$} & $\begin{array}{l}\text { (mg cianidina-3 glu- } \\
\text { cósido/100 g }\end{array}$ \\
\hline Blanco & $34.7 \mathrm{a}^{*}$ & $226.0 \mathrm{a}$ & $495 \mathrm{a}$ & $756 \mathrm{a}$ & $119201 \mathrm{a}$ & $1.33 \mathrm{a}$ \\
\hline Amarillo & $43.6 \mathrm{ab}$ & $242.2 \mathrm{a}$ & $645 \mathrm{a}$ & $1474 \mathrm{a}$ & 100849 a & $0.57 \mathrm{~b}$ \\
\hline Rojo & $38.2 \mathrm{ab}$ & $205.6 \mathrm{~d}$ & 588 a & 1259 a & $128450 \mathrm{a}$ & $9.75 \mathrm{c}$ \\
\hline Azul & $45.5 \mathrm{~b}$ & $220.7 \mathrm{a}$ & $683 \mathrm{a}$ & $1451 \mathrm{a}$ & $127851 \mathrm{a}$ & $6.87 \mathrm{~d}$ \\
\hline Aca & $50.0 \mathrm{~b}$ & $270.1 \mathrm{f}$ & $970 \mathrm{a}$ & $1965 \mathrm{a}$ & 150077 e & $4.63 \mathrm{f}$ \\
\hline \multicolumn{7}{|l|}{ Masa } \\
\hline Blanco & $39.1 \mathrm{ab}$ & $126.1 \mathrm{~b}$ & $7558 \mathrm{~b}$ & $14201 \mathrm{~b}$ & $20470 \mathrm{~b}$ & $0.28 \mathrm{~b}$ \\
\hline Amarillo & $41.5 \mathrm{ab}$ & $140.7 \mathrm{~b}$ & $12596 \mathrm{~d}$ & $20747 \mathrm{cb}$ & $44527 \mathrm{cb}$ & $0.31 \mathrm{~b}$ \\
\hline Rojo & $28.0 \mathrm{c}$ & $97.2 \mathrm{c}$ & $6224 \mathrm{~b}$ & $20747 \mathrm{cb}$ & $22865 \mathrm{~b}$ & $2.21 \mathrm{a}$ \\
\hline Azul & $30.3 \mathrm{ac}$ & $128.2 \mathrm{~b}$ & $10220 \mathrm{bc}$ & $19760 \mathrm{cb}$ & $23330 \mathrm{~b}$ & $2.63 \mathrm{a}$ \\
\hline Aca & $40.3 \mathrm{ab}$ & $158.0 \mathrm{~b}$ & $11170 \mathrm{~b}$ & 24450 c & $40610 \mathrm{cb}$ & $0.56 \mathrm{~b}$ \\
\hline \multicolumn{7}{|l|}{ Tortilla } \\
\hline Blanco & $47.2 \mathrm{~b}$ & $119.0 \mathrm{~b}$ & 9988 c & 23267 c & $51905 c$ & $0.48 \mathrm{~b}$ \\
\hline Amarillo & $51.1 \mathrm{~b}$ & $132.2 \mathrm{~b}$ & $17462 \mathrm{e}$ & 27827 c & $69025 c$ & $0.29 \mathrm{~b}$ \\
\hline Rojo & $30.5 \mathrm{ac}$ & $106.0 \mathrm{c}$ & 8202 bc & $15588 \mathrm{~b}$ & $50036 \mathrm{c}$ & $2.08 \mathrm{a}$ \\
\hline Azul & $39.1 \mathrm{ab}$ & $122.7 \mathrm{bc}$ & 17587 e & $31746 c$ & $52030 \mathrm{c}$ & $3.80 \mathrm{e}$ \\
\hline Aca & $53.0 \mathrm{~b}$ & $154.5 \mathrm{~b}$ & $21566 \mathrm{f}$ & $37743 \mathrm{~d}$ & $77307 \mathrm{c}$ & $0.68 \mathrm{~b}$ \\
\hline \multicolumn{7}{|l|}{ Totopo } \\
\hline Blanco & $46.3 \mathrm{~b}$ & $97.3 \mathrm{c}$ & $6643 \mathrm{~b}$ & 15989 b & $38070 \mathrm{cb}$ & $0.51 \mathrm{~b}$ \\
\hline Amarillo & $43.1 \mathrm{ab}$ & $102.1 \mathrm{c}$ & $13237 \mathrm{~d}$ & 25860 c & $51142 \mathrm{c}$ & $0.36 \mathrm{~b}$ \\
\hline Rojo & $26.4 \mathrm{c}$ & $85.3 \mathrm{e}$ & $8083 \mathrm{bc}$ & 29391 c & $37890 \mathrm{cb}$ & $2.41 \mathrm{a}$ \\
\hline Azul & $41.4 \mathrm{ab}$ & $95.5 \mathrm{c}$ & $14360 \mathrm{~d}$ & 28163 c & $43129 \mathrm{cb}$ & $3.29 \mathrm{e}$ \\
\hline Aca & $46.4 \mathrm{~b}$ & $108.6 \mathrm{c}$ & $13963 \mathrm{~d}$ & 29931 c & $62714 c$ & $0.97 \mathrm{~b}$ \\
\hline
\end{tabular}

Valores con letras distintas dentro de cada hilera son estadísticamente distintos $(\mathrm{P}<0.05) .{ }^{\dagger}$ Modificado de De la Parra et al. $(2007) .{ }^{\dagger \dagger}$ Aca $=$ alto en carotenoides.

luteína en los maíces amarillos, altos en carotenos, y en maíces rojos.

Sin embargo, el procesamiento de la masa en la elaboración de tortillas y totopos no incrementó significativamente las pérdidas. El contenido de zeaxantina fue mayor en los maíces amarillos $(353.2 \mu \mathrm{g} / 100 \mathrm{~g}$ bs), y el de carotenos también fue alto $(322.3 \mu \mathrm{g} / 100 \mathrm{~g} b s)$. Las cantidades de esta xantofila fueron similares en masa, tortillas y totopos para todos los maíces, excepto para el amarillo que tuvo pérdidas durante los pasos subsiguientes de producción de tor- tillas y totopos. Los maíces ricos en carotenos (con granos pigmentados de color naranja, amarillo y rojo) presentaron las mayores cantidades de $\beta$-criptoxantina (23.1, 19.1 y 13.1 $\mu \mathrm{g} / 100 \mathrm{~g}$ bs, respectivamente).

\section{Cambios fitoquímicos y nutracéuticos durante el proceso de extrusión}

La extrusión termoplástica de la harina de maíz en presencia de cal permite la producción alterna de masa o harinas nixtamalizadas. La ventaja de esta tecnología es el 
Cuadro 3. Perfiles de carotenos asociados a maíces criollos blanco, amarillo, rojo, azul, y mejorado alto en carotenos transformados en masas, tortillas y totopos'.

\begin{tabular}{|c|c|c|c|c|}
\hline \multirow{2}{*}{ Tipo de maíz/producto ${ }^{\dagger \dagger}$} & Luteína & Zeaxantina & $\beta$ - Cryptoxantina & $\beta$ - Caroteno \\
\hline & \multicolumn{4}{|c|}{$(\mu \mathrm{g} / 100 \mathrm{~g})$} \\
\hline \multicolumn{5}{|l|}{ Grano } \\
\hline Blanco & $5.73 \mathrm{a}^{\star}$ & $6.01 \mathrm{a}$ & $1.27 \mathrm{a}$ & $4.92 \mathrm{a}$ \\
\hline Amarillo & $406.21 \mathrm{~b}$ & $353.21 \mathrm{~b}$ & $19.10 \mathrm{~b}$ & $33.61 \mathrm{c}$ \\
\hline Rojo & $121.74 \mathrm{c}$ & $111.90 \mathrm{~d}$ & $13.10 \mathrm{c}$ & $20.22 \mathrm{~d}$ \\
\hline Azul & $5.17 \mathrm{a}$ & $14.25 \mathrm{a}$ & $3.41 \mathrm{a}$ & $23.11 \mathrm{~d}$ \\
\hline Aca & $245.63 \mathrm{f}$ & $322.27 \mathrm{~b}$ & $23.11 \mathrm{~d}$ & $45.81 \mathrm{f}$ \\
\hline \multicolumn{5}{|l|}{ Masa } \\
\hline Blanco & $8.16 \mathrm{a}$ & $7.76 \mathrm{a}$ & $1.48 \mathrm{a}$ & nd \\
\hline Amarillo & $129.22 \mathrm{c}$ & $132.42 \mathrm{c}$ & $3.00 \mathrm{a}$ & $20.70 \mathrm{~d}$ \\
\hline Rojo & $42.61 \mathrm{e}$ & $44.86 \mathrm{e}$ & $1.42 \mathrm{a}$ & $7.02 \mathrm{a}$ \\
\hline Azul & $4.71 \mathrm{a}$ & $8.71 \mathrm{a}$ & $1.00 \mathrm{a}$ & $12.50 \mathrm{e}$ \\
\hline Aca & $74.91 \mathrm{~d}$ & $112.61 \mathrm{~d}$ & $11.50 \mathrm{c}$ & $25.92 \mathrm{~d}$ \\
\hline \multicolumn{5}{|l|}{ Tortilla } \\
\hline Blanco & $8.61 \mathrm{a}$ & $7.50 \mathrm{a}$ & $1.57 \mathrm{a}$ & nd \\
\hline Amarillo & $108.21 \mathrm{c}$ & $101.60 \mathrm{~d}$ & $1.81 \mathrm{a}$ & $11.14 \mathrm{e}$ \\
\hline Rojo & $41.40 \mathrm{e}$ & 42.68 ef & $1.32 \mathrm{a}$ & $6.39 \mathrm{a}$ \\
\hline Azul & $4.53 \mathrm{a}$ & $8.81 \mathrm{a}$ & $1.22 \mathrm{a}$ & $12.11 \mathrm{e}$ \\
\hline Aca & $72.51 \mathrm{~d}$ & $105.30 \mathrm{~d}$ & $12.35 \mathrm{c}$ & $14.61 \mathrm{e}$ \\
\hline \multicolumn{5}{|l|}{ Totopos } \\
\hline Blanco & $0.82 \mathrm{a}$ & $9.80 \mathrm{a}$ & $0.08 \mathrm{a}$ & nd \\
\hline Amarillo & $77.27 \mathrm{~d}$ & $64.91 \mathrm{e}$ & $0.11 \mathrm{a}$ & nd \\
\hline Rojo & $40.91 \mathrm{e}$ & 30.16 ef & $1.78 \mathrm{a}$ & nd \\
\hline Azul & $4.48 \mathrm{a}$ & $8.78 \mathrm{a}$ & $0.96 \mathrm{a}$ & nd \\
\hline Aca & $61.12 \mathrm{~d}$ & $92.46 \mathrm{~d}$ & $4.71 \mathrm{a}$ & $8.39 \mathrm{a}$ \\
\hline
\end{tabular}

Valores con letras distintas dentro de cada hilera son estadísticamente distintos $(\mathrm{P}<0.05) .{ }^{\dagger}$ Modificado de De la Parra et al. $(2007) .{ }^{\dagger \dagger}$ Aca, alto en carotenoides.

ahorro en energía, en equipo industrial y en mano de obra. Adicionalmente, esta tecnología permite la elaboración de harinas nixtamalizadas enteras sin producir descargas de "nejayote".

Recientemente, Aguayo-Rojas et al. (2012) estudiaron los perfiles fitoquímicos (fenoles totales y antocianinas) y capacidades antioxidantes (AOX) de cuatro tipos de maíz (blanco, amarillo, rojo y azul) procesados con cal mediante extrusión termoplástica en harinas integrales que luego fueron procesadas en tortillas. La extrusión generó harinas con un contenido mayor $(\mathrm{P}<0.05)$ de fenólicos y antio- xidantes, comparado con las materias primas molidas. De 76.1 a $84.4 \%$ de los fenólicos asociados a los granos y sus tortillas fueron de naturaleza ligada. Las tortillas preparadas con las harinas extrudidas retuvieron de 68.8 a $80.9 \%$ de los fenólicos determinados en los granos enteros. Como se esperaba, el maíz azul presentó las mayores cantidades de antocianinas (27.52 mg cianidina 3-glucósido/100 g bs), cantidad que es 29 veces mayor que en los maíces amarillos, 30 más que en los blancos y 35 más que en los rojos.

Sin embargo, el maíz azul perdió $65 \%$ de sus antocianinas cuando se sometió al proceso de extrusión en presencia 
de cal. La mayor parte de la capacidad antioxidante de los granos crudos (65 a $73 \%$ de la actividad antioxidante) se debió a compuestos ligados. Las tortillas producidas con las harinas extrudidas retuvieron de 67.8 a $75.4 \%$ de sus capacidades antioxidantes determinadas en los granos sin cocer. Los resultados de esta investigación indican claramente que la tecnología de extrusión permite retener a importantes compuestos nutracéuticos que se pierden en el proceso tradicional de producción de tortillas.

\section{USO POTENCIAL DE LOS MAÍCES CRIOLLOS}

Las razas nativas de maíz en México siguen siendo procesadas mediante la nixtamilización, lo cual conlleva a numerosos efectos positivos en la liberación de componentes nutracéuticos, pero también a la degradación y modificación química de otros compuestos nutracéuticos. Esto tiene implicaciones serias en los modelos actuales de mejoramiento de maíces criollos, así como en la producción y procesamiento de alimentos, aspectos que deben considerarse para evitar pérdidas en su valor nutrimental y en su valor benéfico para la salud de los consumidores. En este sentido, las nuevas tecnologías -como la extrusión- que evitan el proceso tradicional de nixtamalización, ayudan a mitigar y salvaguardar el valor nutracéutico de los maíces criollos (de granos azules y rojos), condición que también se observa en las nuevas formas de productos para consumo (granos amarillos) y en la reutilización de algunos productos secundarios con valor agregado, como el "nejayote".

Algunas recomendaciones generadas a partir de este análisis: 1) Promover a nivel del público general las características de interés nutraceútico que los maíces criollos pueden proveer en beneficio de la salud; 2) Hacer que la información generada en diversos centros de investigación sea de conocimiento de la industria, y retroalimentar a la academia con las necesidades de ésta; 3) Proponer de manera puntual esquemas sustentables de cómo potenciar su uso regional y nacional; 4) Difundir la información de la tecnologías de extrusión, sus requisitos técnicos y de adopción del proceso.

Finalmente, debe enfatizarse que las razas nativas en México tienen un potencial enorme aún por descubrir, por lo que se requiere de investigación para salvaguardar la diversidad de germoplasma, que incluye la diversidad nutracéutica y la riqueza culinaria que da origen a una parte fundamental de la alimentación de los mexicanos.

\section{AGRADECIMIENTOS}

A la SAGARPA-CIMMYT, bajo el proyecto "MASAGRO: Modernización Sustentable de la Agricultura Tradicional" y el sub-proyecto "Calidad en Maíces Criollos Mexicanos", por el soporte financiero para la publicación de este manuscrito.

\section{BIBLIOGRAFÍA}

Adom K K, R H Liu (2002) Antioxidant activity of grains. J. Agric. Food Chem. 50:6182-6187.

Aguayo-Rojas J, S Mora-Rochín, E O Cuevas-Rodríguez, S O Serna-Saldívar, J A Gutiérrez-Uribe, C Reyes-Moreno, J Milán-Carrillo (2012) Phytochemicals and antioxidant capacity of tortillas obtained after lime-cooking extrusion process of whole pigmented Mexican maize. Plant Foods Human Nutr. 67:178-185.

Arruzazabala ML, D Carbajal, R Mas, V Molina, S Valdes, A Laguna (1994) Cholesterol lowering effects of polycosanol on rabbits. Biol. Res. 27:205-208.

Arruzazabala M L, S Valdes, R Mas, L Fernández, D Carbajal (1996) Effect of polycosanol successive dose increase on platelet aggregation in healthy volunteers. Pharmacol. Res. 34:181-185.

Betran F J, A J Bockholt, L W Rooney (2001) Blue corn. In: Specialty Corns. A R Hallauer (ed). CRC Press, 2nd ed. Boca Raton, FL., USA. pp:145-169.

Bressani R, R Paz y Paz, N S Scrimshaw (1958) Corn nutrient losses: Chemical changes in corn during preparation of tortillas. J. Agri. Food Chem. 6:770-773.

Chung O K, J B Ohm (2000) Cereal Lipids. In: Handbook of Cereal Science \& Technology. K Kulp, J Ponte (eds). 2nd ed. Marcel Dekker, Inc. NY, USA. pp:56-78.

Cone KC (2007) Anthocyanin synthesis in maize aleurone tissue. Plant Cell Monogr. 8:121-139.

De la Parra C, S O Serna-Saldívar, R H Liu (2007) Effect of processing on the phytochemical profiles and antioxidant activity of corn production of masa, tortillas and tortilla chips. J. Agri. Food Chem. 55:4177-4183.

Del Pozo-Insfran D, C H Brenes, S O Serna Saldívar, S T Talcott (2006) Polyphenolic and antioxidant content of white and blue corn (Zea mays L.) products. Food Res. Int. 39:696-703.

Del Pozo-Insfran D, S O Serna Saldívar, C H Brenes, S T Talcott (2007) Polyphenolics and antioxidant capacity of white and blue corns processed into tortillas and chips. Cereal Chem. 84:162-168.

Egesel C O, J C Wong, R J Lambert, T R Rocheford (2003) Combining ability of maize inbreds for carotenoids and tocopherols. Crop Sci. 43: 818-823.

El-Sayed M, J Abdel-Aal, C Young, I Rabalski (2006) Anthocyanin composition in black, blue, pink, purple, and red cereal grains. J. Agri. Food Chem. 54:4696-4704.

FAO, Food and Agriculture Organization (2012) FAOSTAT Base de Datos Estadísticos. http://faostat.fao.org. Roma, Italia. (Noviembre 2012).

Fiala E S, B S Reddy, J H Weisburger (1985) Naturally occurring anticarcinogenic substances in foodstuffs. Annu. Rev. Nutr. 5:295-321.

Gutiérrez-Uribe J A, C Rojas-García, S García-Lara, S O Serna-Saldívar (2010) Phyto-chemical analysis of wastewater (nejayote) obtained after lime-cooking of different types of maize kernels processed into masa for tortillas. J. Cereal Sci. 52:410-416.

Hagiwara A, K Miyashita, T Nakanishi, M Sano, S Tamano, T Kadota, T Koda, M Nakamura, K Imaida, N Ito, T Shirai (2001) Pronounced inhibition by a natural anthocyanin, purple corn color, of 2-amino-1-methyl-6-phenylimidazo[4,5-b]pyridine (PhIP)-associated colorectal carcinogenesis in male F344 rats pretreated with 1,2-dimethylhydrazine. Cancer Lett. 171:17-25.

Harrigan G G, L G Stork, S G Riordan, T L Reynolds (2007) Impact of genetics and environment on nutritional and metabolite components of maize grain. J. Agri. Food Chem. 55:6177-6185.

Hwang K T, J E Kim, C L Weller (2005) Policosanol contents and composition in wax like materials extracted from selected cereals of Korean origin. Cereal Chem. 82:242-245.

Kornfeldt A, L B Croon (1981) 4-Demethyl-, 4-monomethyl- and 4,4-dimethylsterols in some vegetable oils. Lipids 16:306-314.

López-Martínez L X, K L Parkin, H S García (2011) Phase II-inducing, polyphenols content and antioxidant capacity of corn (Zea mays L.) from phenotypes of white, blue, red and purple colors processed into masa and tortillas. Plant Foods Human Nutr. 66:41-47. 
Mora-Rochín S, J A Gutiérrez-Uribe, S O Serna-Saldívar, P SánchezPeña, C Reyes-Moreno, J Milán-Carrillo (2010) Phenolic content and antioxidant activity of tortillas produced from pigmented corns processed by conventional nixtamalization or extrusion cooking. J. Cereal Sci. 52:502-508.

Ortega P R A, J J Sánchez G, F Castillo G, J M Hernández C (1991) Estado actual de los estudios sobre maíces nativos en México. In: Avances en el Estudio de los Recursos Fitogenéticos de México. P R A Ortega, G Palomino H, F Castillo G, V A González H, M Livera M (eds). SOMEFI. Chapingo, México. pp:161-185.

Pedreschi R, L Cisneros-Zevallos (2007) Phenolic profiles of Andean purple corn (Zea mays L.). Food Chem. 100:956-963.

Prochaska H J, P Talalay (1988) Regulatory mechanism of monofunctional and bifuctional anticarcinogenic enzyme inducers in murine liver. Cancer Res. 48:4776-4782.

Raicht R F, B I Cohen, E P Fazzini, A N Sarwal, M Takahashi (1980) Protective effect of plant sterols against chemically induced colon tumors in rats. Cancer Res. 40:403-405.

Salinas-Moreno Y, F Martínez-Bustos, M Soto-Hernández, R OrtegaPaczka, J L Arellano-Vázquez (2003) Efecto de la nixtamalización sobre las antocianinas del grano de maíces pigmentados. Agrociencia 37:617-628.

Serna-Saldívar S O, S García Lara, J A Gutiérrez Uribe (2011) Perfil fitoquímico y propiedades nutracéuticas de maíces pigmentados y sus tortillas. In: Amplitud, Mejoramiento, Usos y Riesgos de la Diversidad Genética de Maíz en México. R E Preciado Ortiz, S Montes Hernández (eds). Sociedad Mexicana de Fitogenética AC, Chapingo, Estado de México, México. pp:75-96.

Serna-Saldívar S O, M H Gómez, L W Rooney (1990) Technology, chemistry and nutritional value of alkaline cooked corn products.
In: Advances in Cereal Sci. \& Technology. Y Pomeranz (ed). American Association of Cereal Chemists, St. Paul, MN, USA. Vol. X. pp: 245-265.

Serna-Saldívar S O (2009) Research developments in the science, technology and nutritional value of maize-based nixtamalized foods. In: The ICC Book of Ethnic Cereal-Based Foods Across the Continents. J Taylor, R Cracknell (eds). Published by the University of Pretoria, Pretoria, South Africa. pp:133-163.

Serna-Saldívar S O (2010) Cereal Grains: Properties, Processing, and Nutritional Attributes. CRC Press, Boca Raton, FL, USA. 747 p.

Shih P H, C T Yeh, G C Yen (2005) Effects of anthocyanidin on the inhibition of proliferation and induction of apoptosis in human gastric adenocarcinoma cells. Food Chem.l Toxicol. 55:1557-1566.

Steer T E, G R Gibson (2002) The microbiology of phytic acid metabolism by gut bacteria and relevance for bowel cancer. Int. J. Food Sci. Technol. 37:783-790.

Tsuda T, F Horio, K Uchida, H Aoki, T Osawa (2003) Dietary cyanidin 3-O- $\beta$-D-glucoside-rich purple corn color prevents obesity and ameliorates hyperglycemia in mice. Nutrient-Gene Interact. 314:2125-2130.

Wang L S, G D Stoner (2008) Anthocyanins and their role in cancer prevention. Cancer Lett. 269:281-290.

Warburton M L, J C Reif, M Frisch, M Bohn, C Bedoya, X C Xia, J Crossa, J Franco, D Hoisington, K Pixley, S Taba, A E Melchinger (2008) Trends in genetic diversity in CIMMYT non-temperate maize germplasm. Crop Sci. 48:617-624.

White P, E J Weber (2003) Lipids of the kernel. In: Corn Chemistry and Technology. P White, L Johnson (eds). American Association of Cereal Chemists, Second Edition. St. Paul, MN, USA. pp:3948. 